Jpn. J. Med. Sci. Biol., 45, 87 - 97, 1992.

\title{
The Second NIH Symposium
}

on Molecular Biology of Hepatitis Viruses, held on February 13, 1992

Opening Remarks. Tohru Tokunaga (Director-General, NIH)

Session I. Introduction

Chairman: Takashi Kitamura (NIH)

1. Hepatitis $\mathrm{C}$ virus infection and hepatocellular carcinoma: Kusuya NISHIOKA

Session II. A type and E type Hepatitis viruses

Chairman: Hiroshi Yoshikura (NIH / Univ. of Tokyo)

1. Molecular features of HEV genome: Daniel W. BRADLEY

2. Molecular epidemiology of hepatitis A virus in Japan: Yasuo MORITSUGU

Session III. B type hepatitis virus

Chairman: Takeshi Kurata (NIH)

1. Enhanced virulence of hepatitis B virus with mutations at specific sites within the precore region: Shunji MISHIRO

2. Pathobiological implications of genetic heterogeneity of pre- $\mathrm{C} / \mathrm{C}$ region of HBV-DNA: Ferruccio BONINO

3. Hepatitis B virus $X$ gene and hepatocellular carcinoma: Kazuhiko KOIKE

Session IV. C type hepatitis virus

Chairman: Tatsuo Miyamura (NIH)

1. Structure of the type $\mathrm{C}$ hepatitis virus (HCV) genome: Nobuyuki KATO

2. Gene expression of HCV structural proteins and their applications: Izumu SAITO

3. Treatment of chronic hepatitis $\mathrm{C}$ with interferons: Shiro IINO

Panel Discussion by all speakers

Chairmen: Yasuo Moritsugu (NIH), Tatsuo Miyamura (NIH)

Closing Remarks. Shudo Yamazaki (Deputy Director-General, NIH) 


\section{HEPATITIS C VIRUS INFECTION AND HEPATOCELLULAR CARCINOMA}

Kusuya NISHIOKA

The Japanese Red Cross, Central Blood Center

Hepatocellular carcinoma (HCC) has been recognized as one of the most prevalent and most lethal cancers in the countries of Asia, Africa, Oceania, Southern Europe and Latin American countries. The epidemiological and clinical studies based on immunology and molecular biology in defined population provided important answers to etiology of this cancer. Not only persistent hepatitis $\mathrm{B}$ virus (HBV) infection hitherto reported, but also hepatitis $\mathrm{C}$ virus (HCV) infection play important etiological roles in a great majority of HCC showing $30-70 \%$ positivity of anti-HCV among HCC patients in Asian countries. They are preventable by control measures for these types of viral infection. Anti$\mathrm{HCV}$ positive rate in $\mathrm{HBsAg}$ positive cases are $30-50 \%$ in HCC patients as compared with 1 to $2 \%$ in healthy blood donors or in asymptomatic $\mathrm{HBsAg}$ carriers. This indicates that double infection of $\mathrm{HBV}$ and $\mathrm{HCV}$ is extremely high risk for development of $\mathrm{HCC}$ in $\mathrm{HBV}$ endemic areas.

Blood transfusion has been documented as a major transmission route of $\mathrm{HCV}$ which related to development of HCC afterwards. Since anti-HCV screening of donated blood is extremely effective for prevention of posttransfusion $\mathrm{HCV}$ infection, it is especially important to prevent $\mathrm{HCV}$ infection in $\mathrm{HCV}$ endemic areas by screening of anti-HCV positive blood for transfusion in these areas.

\section{MOLECULAR FEATURES OF HEV GENOME}

Daniel W. BLADLEY

Hepatitis Branch, U. S. Centers for Disease Control

\section{Molecular cloning of $\mathrm{HEV}$}

Hepatitis E virus (HEV), the major agent of enterically transmitted non-A, non-B hepatitis (ET-NANBH), is a $32 \mathrm{~nm}$ diameter, non-enveloped virus with 
physicochemical properties similar to many well-characterized caliciviruses or calici-like viruses. HEV has a sedimentation coefficient of $183 \mathrm{~S}$ in sucrose and is extremely labile in the presence of high salt concentration. Cynomolgus macaques (cynos) have been shown to be susceptible to infection with human $\mathrm{HEV}$ and have been extensively used for the generation of virus-positive source materials and antisera reactive with HEV particles and antigen(s). Molecular cloning of HEV was approached with the assumption that it had an RNA genome. Molecular clones of HEV were identified by two separate procedures using lambda gt10 and gt11 as cloning and expression vectors, respectively. cDNA was prepared from both uninfected and HEV infected cyno bile (Burma isolate) and inserted into lambda gt10 for "plus-minus" screening using cDNA probes (from both cDNA libraries) labeled by random priming with 32P NTPS. Differential hybridization studies, using radiolabeled probes, revealed the presence of several candidate clones derived from infected cyno bile, including ET1.1, the first virusspecific clone to be identified. ET1.1 was shown to, (1) hybridize specifically to infected source cDNA, (2) hybridize specifically to infected liver RNA (approx. 7.5 $\mathrm{Kb}$ polyadenylated molecules), encode a portion of an RNA-dependent RNA polymerase consensus motif, and (4) be exogenous to both infected and uninfected primate DNA. Clone ET1.1 was also shown to specifically hybridize to cDNAs prepared from other HEV-infected stools collected from outbreak-related cases in Borneo, Mexico, Pakistan, Somalia, and the USSR. The latter cDNAs were prepared by a novel cDNA amplification procedure (SISPA) that permitted the expansion of the limited amount of cDNA originally prepared from the source stools.

The second approach to HEV clone identification relied on the use of immunoscreening of cDNA libraries (prepared from a Mexican outbreak case stool) prepared in lambda gt11, a highly efficient expression vector. Virusspecific proteins, expressed as betagalactosidase fusion proteins, were identified using convalescent serum from another well-documented case of hepatitis $\mathrm{E}$ from a separate outbreak in Mexico. Two of these virus-specific clones, 406.3-2 and 406.4-2, were used to identify additional virus-specific messages of 2.0 and $3.7 \mathrm{~Kb}$ in polyA-RNA extracted from HEV infected cyno liver, indicating the possible presence of subgenomic strands required for virus replication. Paneling of expressed proteins against a variety of paired acute and convalescent phase human sera from geographically distinct regions of the world, as well as preinoculation, acute-phase, and convalescent-phase sera from infected cynos, demonstrated the specificity of these expressed proteins for hepatitis $E$. 


\section{Genomic organization}

Analysis of the entire nucleotide sequence of the HEV-Burma isolate has revealed the presence of several consensus sequences, including one associated with an NTP-binding, helicase-like function 5' to the putative RNA-dependent RNA polymerase (RDRP). These nonstructural elements are contained within a single open reading frame (ORF) of approximately $5 \mathrm{~Kb}$ beginning 27 nucleotides from the 5' end of the genome. Clone 406.3-2 was contained within a second ORF that extended another $2 \mathrm{~Kb}$ to a point $68 \mathrm{~b}$ upstream from the 3 ' polyA tail. ORF2 is thought to encode for the major structural protein(s) of HEV. The second immunoreactive clone, 406.4-2, was identified within a third ORF (plus 2 frame) that contained only 369 nucleotides. It overlapped ORF 1 by one nucleotide and ORF2 by 328 nucleotides. HEV appears to be substantially different from picornaviruses, including $\mathrm{HAV}$, in that it has an RNA genome that encodes for putative structural and nonstructural proteins through the use of discontinuous, partially overlapping ORFs.

The genomes of both Burma and Mexico HEV isolates have now been cloned and completely sequenced; they have been shown to possess the basic genomic features described above. Comparison of the nucleotide sequences of Burma, Mexico, Pakistan, Chinese (PRC), and USSR isolates within the region encoding for the putative RDRP show a 94\% homology between all but the Mexican isolate, which shares a 77\% homology with each of the other isolates. Conservation at the deduced amino acid level is approximately $96 \%$ and $89 \%$, respectively, for the above isolates. We believe that a basic understanding of the key elements of the genetic organization and expression strategy of HEV (still tentative, pending sequencing of authentic viral proteins) has been achieved and shown to set it apart from other previously defined hepatotropic viruses of man. Recent studies of the genome of Norwalk virus (M. K. Estes, personal communication) further suggest that HEV may belong to a larger family of single-stranded, polyadenylated RNA viruses that have multiple ORFs and replicate via the use of defined-length, subgenomic elements. 


\title{
MOLECULAR EPIDEMIOLOGY OF HEPATITIS A VIRUS IN JAPAN
}

\author{
Yasuo MORITSUGU \\ Department of Enteroviruses, National Institute of Health (Murayama Annex)
}

Human hepatitis A virus (HAV), a unique hepatotropic member of the picornavirus family, has been recently reclassified in the new genus, Hepatovirus, since genomic sequences of the virus has no similarity with entero or rhinoviruses. HAV is antigenically strongly conserved and the strains are indistinguishable by serological means not only with polyclonal antibodies elicited by either natural or experimental HAV infections but also with monoclonal antibodies raised against various HAV strains. However, recent studies of the nucleotide sequences have demonstrated substantial heterogeneity among individual HAV strains. HAV strains have been proposed to be classified into genotypes by the comparison of 168 nucleotide sequences in the putative VP1/2A junction region.

The usefulness of the nucleotide sequencing in HAV strain identification was confirmed employing cDNA products amplified by the PCR technique. A total of 14 stool samples were used in the study. Eleven specimens were collected from patients involved in a school outbreak and three were collected from sporadic cases of hepatitis A. The stability of the HAV sequence was confirmed by the tests with eight cell culture isolates and a monkey isolate, and later reconfirmed by the serial stool samples obtained from individual cases over a period of time.

Including the above 14 samples, a total of 119 cDNA samples were amplified by the PCR technique from stools collected from 111 hepatitis A cases occurred in Japan. Among them, 14 specimens were obtained from six patients by serial collection, and among the 111 individual cDNA samples, seven contained mixed sequences. The nucleotide variations of 104 strains were compared within a 168 base region of the putative VP1/2A cleavage site, and were identified by the unique sequence into 22 variants of genotype IA, one variant of genotype IB and three variants of genotype IIIB. 
ENHANCED VIRULENCE OF HEPATITIS B VIRUS WITH MUTATIONS AT SPECIFIC SITES WITHIN THE PRECORE REGION

\author{
Shunji MISHIRO \\ Institute of Immunology, and The Kitasato Institute, Tokyo
}

Genotype-phenotype correlations are a recent subject of hepatitis B virus (HBV) research. A precore-defective HBV variant, which cannot produce HBeantigen because of a G-to-A mutation within the precore gene at -5 position preceding the AUG initiator for core protein, has been implicated in many cases of severe, often fulminant, hepatitis, with speculations that HBe-antigen may modulate the immune response of the host. Our present study suggests that another G-to-A mutation at the -2 position, which does not lead to precore deficiency, may also enhance $\mathrm{HBV}$ virulence. A possible mechanism is, as rehearsed by Hasegawa et al., that the G-to-A mutation at either or both the $-5,-2$ positions induces the precore/core junction sequence to form a stem-loop conformation which favors encapsidation of the HBV pregenome into core particles. This mechanism may also explain why such variants overgrow preexisting wild type $\mathrm{HBV}$ during the course of persistent infection.

\title{
PATHOBIOLOGICAL IMPLICATIONS OF GENETIC HETERO- GENEITY OF Pre-C/C REGION OF HBV-DNA
}

Ferruccio BONINO and Maurizia Rossana BRUNETTO

Hepatology and Laboratory Units, Division of Gastroenterology, Molinette Hospital, Italy

Hepatitis B virus (HBV) variants unable to secrete hepatitis B "e" antigen ( $\mathrm{HBeAg})$ explain the serological pattern of patients with chronic anti-HBe positive hepatitis $B$, originally described in the Mediterranean area. In these patients persistence serum HBV-DNA and intrahepatic hepatitis B core antigen, $\mathrm{HBcAg}$ is associated with the infection of $\mathrm{HBV}$ mutants, defective in the expression of the pre-core region of the $\mathrm{C}$ gene that is necessary for secretion of 
HBeAg. A mutation from guanosine (G) to adenosine (A) at nucleotide 1896 of pre-core region, resulting in a translational stop codon was found as the most frequent cause (in more $90 \%$ of cases) of defective expression of pre-core. This mutation does not alter the in vitro and vivo viability of HBV. Other mutations such as nonfunctional start codons (conversion of ATG into ATA, AGG, TTG or ATT), nucleotide insertions or deletions resulting in frame shifts or other stop codons (TAA at nucleotide 1817) were described, but at a very low frequency. The $\mathrm{HBeAg}$ minus mutant (nucleotide 1896) has been found in unrelated patients of Italy, Greece, Bulgaria, Turkey, Japan, China, Indonesia and Kenya, suggesting that it is associated with chronic anti-HBe positive hepatitis B worldwide and not segregated in restricted geographical areas as originally believed. Anti-HBe positive hepatitis B shows a more severe course than classical $\mathrm{HBeAg}$ positive hepatitis, uncommon spontaneous remissions and a slow rate of response to interferon. In addition $\mathrm{HBeAg}$ minus $\mathrm{HBV}$ prevails during the $\mathrm{HBeAg} /$ anti-HBe seroconversion phase and is fulminant hepatitis $B$. These findings suggest that immunoelimination determines a positive selection of $\mathrm{HBeAg}$ minus $\mathrm{HBV}$. However, we found $\mathrm{HBeAg}$ minus $\mathrm{HBV}$ in any phase of HBV infection: in $27 \%$ of immunotolerant HBV carriers, in $67 \%$ of patients with chronic hepatitis B (immunoelimination phase) and in $17 \%$ of $\mathrm{HBsAg}$ carriers with latent $\mathrm{HBV}$ infection. Therefore the early sequence of $\mathrm{HBeAg}$ minus $\mathrm{HBV}$ during the immunotolerance phase suggests that its presence can be independent from the pressure of immune system. We found that $\mathrm{HBeAg}$ minus viremia increases significantly after ALT flare-ups while wild-type viremia increases before episodes of liver cell necrosis. In addition a relative $\mathrm{HBeAg}$ defective viremia higher than $20 \%$ at baseline was shown as a highly statistically significant predictor of non-response to interferon. Positive selection of the defective virus with liver disease and its vulnerability by the immune system and interferon significantly lower than wild-type virus are intriguing and apparently in contradiction. However, considering that "e" antigen contributes to immunotolerance, we may speculate that $\mathrm{HBeAg}$ defective $\mathrm{HBV}$ can contribute to lower "e" antigenemia below a critical level that maintains tolerance. In conclusion, the $\mathrm{G}$ to $\mathrm{A}$ switch at base 1896 of the HBV-DNA pre-core region appears a special strategy of HBV to survive under special conditions rather than an occasional urgency of an escape mutant. The mechanisms that regulate transition from immunotolerance to immunoelimination remain to be clarified as well as the reasons of the extraordinary prevalence of this mutation over all the others capable to preclude secretion of $\mathrm{HBeAg}$. 


\section{HEPATITIS B VIRUS X GENE AND HEPATOCELLULAR}

\section{CARCINOMA}

\section{Kazuhiko KOIKE}

First Department of Internal Medicine, University of Tokyo

The exact role hepatitis B virus in the development of hepatic cancer is not clear. The recent identification of a viral regulatory gene $\mathrm{X}$ suggests a possible direct involvement of the virus, whereby the $\mathrm{X}$ protein may alter host gene expression and lead to the development of hepatocellular carcinoma. We have tested this possibility by placing the entire $\mathrm{X}$ gene under its own regulatory elements directly into the germline of mice. Transgenic mice carrying this vial gene succumbed to progressive histopathological changes specifically in the liver, beginning with multifocal areas of altered hepatocytes, followed by the appearances of benign adenomas, and proceeding to the development of malignant carcinomas. These results show, for the first time, the direct involvement of hepatitis B virus in the development of liver cancer. Molecular events that follow the expression of the $\mathrm{X}$ gene in mouse liver were studied by analyses of gene rearrangement, oncogene expression, histopathological examination etc. These results will be discussed. 


\title{
STRUCTURE OF THE TYPE C HEPATITIS VIRUS (HCV) GENOME
}

\author{
Nobuyuki KATO, Makoto HIJKATA, Torahiko TANAKA, Myung-Je CHO, \\ Shigehisa MORI and Kunitada SHIMOTOHNO
}

\section{Virology Division, National Cancer Research Institute}

Hepatitis $\mathrm{C}$ virus (HCV) is the major causative agent of post-transfusional non-A, non-B hepatitis throughout the world. We have determined the nucleotide sequence of the entire Japanese type of HCV (HCV-J) genome, which contains a long open reading frame that can encode a sequence of 3010 amino acid residues. This structural analysis revealed that HCV belongs to a new genus distinct from the flaviviruses and pestiviruses of the Flaviviridae. Furthermore, the nucleotide sequences of two entire viral genomes (HCV-JT and HCV-J33), which belong to HCV-J genotype, were also determined and we found the sequence diversity among the isolates of HCV-J genotype (average $8 \%$ in nucleotide sequence and $5 \%$ in amino acid sequence). Marked sequence diversity among the virus isolates was found in the putative envelope region and two hypervariable regions (HVR1 and HVR2) were found in this region. HVR2 was present in HCV-J isolates, but not in American type of HCV (HCV-US) isolates.

We examined the distribution of HCV genotypes (HCV-J, HCV-US, HCV$\mathrm{K} 2 \mathrm{a}$ and $-\mathrm{K} 2 \mathrm{~b}$ ) in Japan and obtained the evidence that HCV-J was a dominant $\mathrm{HCV}$ genotype in Japan. In these typing analysis, we detected new HCV genotypes (HCV-Ta and -Tb) from Thai patients.

Processing of the proteins encoded in HCV-J genome was examined by using an in vitro expression system. We found three products which were arranged from the amino-terminal end of the polyprotein precursor in the order: NH2-p22gp35-gp70, as the putative structural proteins. Furthermore, three additional products, $\mathrm{p} 21, \mathrm{p} 70$ and $\mathrm{p} 30$ following to gp70 were also found in the order: $\mathrm{p} 21$ p70-p30. p70 was shown to have proteinase activity in vitro processing system. 
GENE EXPRESSION OF HCV STRUCTURAL PROTEINS AND THEIR APPLICATIONS

\author{
Izumu SAITO, Shizuko HARADA1, Yoshiharu MATSUURA2, \\ Yoshimichi INOUE3, Ryosuke SUZUKI ${ }^{3}$, Ayako ANDO3, \\ Don-Wan KIM33, Yushiro WATANABE4, Joe CHIBA5 and \\ Tatsuo MIYAMURA3
}

Laboratory of Molecular Genetics, Institute of Medical Science, University of Tokyo, Departments of 1Medical Entomology, 2Veterinary Science and 3 Enteroviruses, National Institute of Health, 4 St. Marianna University School of Medicine, and 5Science University of Tokyo

HCV core protein, named p22, was expressed both in COS cells and in insect cells by using baculovirus vector. A practical ELISA system for detection of HCV core antibody was established using p22 expressed in insect cells. The detection system was found valuable for early diagnosis of hepatitis $\mathrm{C}$ and screening of blood for transfusion. A c-terminally truncated form of $\mathrm{p} 22$, originally made by intending easy purification, was found unexpectedly to be located in nuclei when expressed in cultured cells. HCV core fusion proteins with either human serum albumin or apolipoprotein $\mathrm{E}$ were expressed but so far none of the fusion proteins we made were secreted into culture medium. HCV envelope (env) and NS1 proteins were expressed both in insect cells by using baculovirus vector and in $\mathrm{CHO}$ cells by using mulcos expression vector (Gene, 71: 19-27, 1988). By Western blot analysis, HCV env antibody was suggested to be possible neutralization antibody. HCV NS1 antibody is detected in $90 \%$ of the patient sera of chronic non-A, non-B hepatitis, showing that the NS1 antibody test is also valuable for diagnosis of hepatitis $\mathrm{C}$. 


\section{TREATMENT OF CHRONIC HEPATITIS C WITH INTERFERONS}

Shiro IINO

First Department of Internal Medicine, The University of Tokyo

Since Hoofnagle first reported that Interferon (IFN) administration on nonA, non-B chronic hepatitis (NANBCH) decreased alanine aminotransferase (ALT) activity, and the long-term decline in ALT activity in due course, improved histological findings of the liver. IFN has been introduced in the treatment of chronic hepatitis $\mathrm{C}$ (CHC).

Afterwards, IFN has been used worldwidely in the treatment of CHC. In case of IFN- $\alpha$, most of the regimen is 3 to 6 million international units (MIU) of IFN administered three times a week for 6 to 12 months. With this regimen, however, although ALT levels are declined or normalized during IFN treatment, re-elevation of ALT levels after cessation of treatment is observed at high frequencies, which remains a critical problem to be solved. In these circumstances, we conducted a comparative study, giving different doses of IFN- $\beta$ daily for 8 consecutive weeks. Consequently we showed normalization of ALT levels were achieved at high frequencies after discontinuation of treatment in patients treated with $6 \mathrm{MIU}$ of IFN- $\beta$, which attracted considerable attention.

In Japan, thereafter, the target of IFN treatment was placed on to increase the rate of ALT normalization after therapy, and large-scale comparative studies using various IFNs have been conducted with regimens based on the above described 8-week consecutive administration, which produced fruitful results.

I will present three data and discuss the present and future problems on IFN therapy of chronic hepatitis $\mathrm{C}$. 